\title{
Photoelectron angular distributions from resonant two-photon ionization of adiabatically aligned naphthalene and aniline molecules
}

\author{
Jacqueline Arlt, ${ }^{1}$ Dhirendra P. Singh, ${ }^{2}$ James O. F. Thompson, ${ }^{2}$ Adam S. Chatterley, ${ }^{1}$ Paul \\ Hockett, ${ }^{3}$ Henrik Stapelfeldt ${ }^{1 *} \&$ Katharine L. Reid ${ }^{2 *}$ \\ 1 - Department of Chemistry, Aarhus University, 8000 Aarhus C, Denmark \\ 2 - School of Chemistry, University of Nottingham, Nottingham, NG7 2RD, UK \\ 3 - National Research Council of Canada, 100 Sussex Drive, Ottawa, K1A 0R6, Canada \\ *Corresponding Authors
}

\section{Abstract}

Photoelectron images have been measured following the ionization of aligned distributions of gas phase naphthalene and aniline molecules. Alignment in the adiabatic regime was achieved by interaction with a 100 ps infrared laser pulse, with ionization achieved in a two-photon resonant scheme using a low intensity UV pulse of $\sim 6$ ps duration. The resulting images are found to exhibit anisotropy that increases when the alignment pulse is present, with the aniline PADs peaking along the polarization vector of the ionizing light and the naphthalene PADs developing a characteristic four-lobed structure. Photoelectron angular distributions (PADs) that result from the ionization of unaligned and fully aligned distributions of molecules are calculated using the ePolyScat ab initio suite and converted into two-dimensional photoelectron images. In the case of naphthalene excellent agreement is observed between experiment and the simulation for the fully aligned distribution, showing that the alignment step allows us to probe the molecular frame, but in the case of aniline it is clear that additional processes occur following the one-photon resonant step. 


\section{Introduction}

Photoelectron angular distributions (PADs) are uniquely sensitive to electronic structure and dynamics, to nuclear configuration and dynamics, and to the chirality and conformation of isolated complex systems such as biomolecules and supra-molecular assemblies. ${ }^{1,2}$ PADs can be measured in a variety of experimental scenarios, dictating the information that can be gleaned. Of particular interest have been techniques that enable PADs to be measured "from the point of view of a molecule"; so-called molecular frame (MF) PADs. ${ }^{1,2}$ Although rich information on photoionization dynamics can be achieved for light polyatomic molecular systems by exploiting polarization geometries in multiphoton ionization experiments, ${ }^{3}$ MFPADs provide a means of obtaining comparable information on the photoionization dynamics of heavier and more complex molecular systems.

There are two main techniques that have been used to access MFPADs. The first of these relies on a dissociative ionization process in which the emitted photoelectron is detected in coincidence with one or more ionic fragments. ${ }^{4-9}$ This technique requires either the availability of dissociative ionization pathways in which fragments are ejected in the axial recoil limit or the use of an intense electric field to fragment the molecule in a Coulomb explosion. The second technique, employed in this work, uses a moderately strong laser field to create an aligned distribution of molecules prior to ionization. ${ }^{10}$ Alignment can be achieved in either one dimension (confinement of a single molecular axis in the laboratory frame) or three dimensions (confinement of all three principal molecular axes in the laboratory frame). The alignment is generally characterized by the value of $\left\langle\cos ^{2} \theta_{\widehat{n}}\right\rangle$, where $\theta_{\hat{n}}$ is the angle between the polarization vector of the alignment pulse and the molecular axis being considered. Using this terminology, $\left\langle\cos ^{2} \theta_{\widehat{n}}\right\rangle=1$ represents complete alignment (coincidence of the molecular frame and laboratory frame axes) and $\left\langle\cos ^{2} \theta_{\widehat{n}}\right\rangle=1 / 3$ represents an isotropic distribution. The measured PADs will converge on the molecular frame if the degree of alignment is very high 
(typically $\left\langle\cos ^{2} \theta_{\widehat{n}}\right\rangle>0.9 .{ }^{11}$ The technique applies to most molecules that can be delivered in a cold molecular beam, as long as they possess an anisotropic polarizability tensor.

Laser-induced alignment falls into two limiting regimes; the nonadiabatic and the adiabatic. In the nonadiabatic regime, alignment is induced by a laser pulse, which is short with respect to the molecular rotational period(s), or by a rapidly truncated pulse of longer duration. The rotational wave packets thus created lead to molecular alignment in regularly spaced, narrow time windows termed revivals. A major advantage of the revivals is that they occur after the laser pulse is turned off, such that the influence of the laser field on any observed dynamics of the aligned molecules is eliminated. Photoelectron angular distributions measured from aligned molecules under the fieldfree conditions provided by rotational revivals have been used to explore strong-field ionization, ${ }^{12}$ femtosecond time-resolved photodissociation, ${ }^{13}$ molecular orbital dynamics, ${ }^{14}$ and single photon ionization with VUV pulses. ${ }^{15-18}$ However, revivals typically provide efficient alignment for only a few hundred femtoseconds, which prevents observations of the dynamics of longer-lived molecular processes. Furthermore, a high degree of alignment at a revival is normally only possible for linear and symmetric top molecules, and the best alignment requires pulse-shaping and quantum state selection. ${ }^{19}$ For asymmetric top molecules, the irregular rotational level structure hampers sharp alignment at revivals ${ }^{20}$ and, in particular, makes it challenging to achieve efficient field-free 3D alignment. ${ }^{21-23}$

The adiabatic alignment regime is realized by turning on the alignment pulse slowly with respect to molecular rotation. In this way, the degree of alignment follows the intensity profile of the pulse and therefore reaches a maximum at the peak of the pulse. Here, it can be very high, approaching true fixed-in-space conditions. Adiabatic alignment applies to a broad range of molecules; it lasts as long as the pulse is on and it is straightforward to implement 3D alignment. ${ }^{24}$ Furthermore, it is possible to achieve molecular orientation in which the direction of a permanent dipole moment in a polar molecule is defined. ${ }^{25}$ These advantages of adiabatic alignment have been exploited in 
measurements of PADs following strong-field ionization, which have been used to image nodal planes in molecular orbitals, ${ }^{26,27}$ to explore low energy structures with elliptically polarized pulses, ${ }^{28}$ and to demonstrate that 3D MFPADS can be obtained by controlled molecular rotation in combination with tomographic reconstruction methods. ${ }^{29}$ PADs measured following ionization of adiabatically aligned molecules with $\mathrm{x}$-ray pulses, which are aimed at structural imaging through electron diffraction, have also been reported. ${ }^{30}$ The advantages of adiabatic alignment, however, come at a price, namely that the alignment occurs in the presence of the laser field. For the strongfield ionization studies referred to above, this was not observed to cause significant complications, but molecules in electronically excited states are likely to be perturbed by an alignment pulse whose intensity is $>10^{11} \mathrm{~W} / \mathrm{cm}^{2}$. In the present work, we study weak-field ionization of electronically excited naphthalene and aniline that have been adiabatically aligned, and in the case of aniline only we observe unwanted complications from the alignment pulse.

In what follows, we present experimental measurements of photoelectron images following twophoton ionization of adiabatically aligned naphthalene and aniline molecules. In both cases, the twophoton process is resonant at the one-photon level with the $S_{1}$ electronically excited state. In order to interpret these results, we present numerical simulations that project limiting case PADs, computed using ab initio scattering calculations, into two-dimensional photoelectron images.

\section{Experimental Method}

Schematics of the key elements in the experimental set-up are given in Figs. 1a and 1b. More details can be found in Ref. 31. A molecular beam is formed by expanding a gas mixture of 80 bar of helium and 2 mbar of aniline or $1 \mathrm{mbar}$ of naphthalene into a vacuum chamber through an Even-Lavie valve EL-7-4-2015-HRR,HT. ${ }^{32}$ In the case of aniline (naphthalene), the valve was heated to $36^{\circ} \mathrm{C}\left(50^{\circ} \mathrm{C}\right)$. After passage through a 4-mm diameter skimmer, the molecular beam enters a velocity map imaging (VMI) spectrometer where it is crossed at $90^{\circ}$ by two collinearly focused, pulsed laser beams, one to align the molecules and one to ionize them. The electrons produced by the ionization 
pulse are extracted with the static electric field $(140-150 \mathrm{~V} / \mathrm{cm})$ in the VMI spectrometer, and projected onto a two-dimensional imaging detector consisting of a microchannel plate detector with an active diameter of $41.5 \mathrm{~mm}$ backed by a phosphor screen; see Fig. 1(b). The electron hits on the detector are recorded by a CCD camera (Prosilica GE 680, Allied Vision) and on-line software analysis determines and saves the coordinates of each individual electron hit. Thus, the basic experimental observables are two-dimensional velocity images of the photoelectrons. The repetition rate of the experiment is $200 \mathrm{~Hz}$, limited by the pump speed in the vacuum chamber for the Even Lavie valve.

A schematic of the laser set-up is shown in Fig. 1a. Both laser beams originate from the same pulsed Ti:Sapphire laser system (customized Solstice ACE, model 80L-35F-1K-HP-T Spectra-Physics, 1 kHz, $6 \mathrm{~W}, 40 \mathrm{fs})$. A part of the uncompressed $800 \mathrm{~nm}$ output of the laser system provides the alignment beam. These laser pulses have a duration of 110 ps (FWHM, see Fig. 1c for pulse profile) and a pulse energy up to $700 \mu \mathrm{J}$. The beam used to ionize the molecules is obtained from an optical parametric amplifier (TOPAS, model TP8U1, Light Conversion, pumped by $3 \mathrm{~mJ}$ from the compressed output of the Ti:Sapphire laser system) and subsequent nonlinear optical processes. The TOPAS idler output at $\sim 2215 \mathrm{~nm}$ is frequency-mixed with an $800 \mathrm{~nm}$ beam from the Ti-Sapphire laser in a 0.5-mm thick BBO crystal to generate a visible beam at $\sim 588 \mathrm{~nm}$.

This beam is then frequency doubled in $40-\mathrm{mm}$ thick KDP crystal to yield the final UV probe beam, which is linearly polarized. The intensity profile of the probe pulse, obtained by cross correlation with a 40 -fs-long $800 \mathrm{~nm}$ pulse, is shown in the inset of Fig. 1c. The width at half maximum of the pulse is $0.6 \mathrm{ps}$ but there is a long tail extending to $\sim 5 \mathrm{ps}$. This asymmetric shape as well as the bandwidth of the probe pulse, $\sim 0.06 \mathrm{~nm}$, is due to the thick KDP crystal used for the frequency doubling.

For the aniline measurements, the pulses were centered at $293.85 \mathrm{~nm}$, and the pulse energy was up to $0.2 \mu \mathrm{J}$. For the naphthalene measurements, the pulses were centered at $293.53 \mathrm{~nm}$ and the pulse energy was $2 \mu \mathrm{J}$. 
A dichroic mirror (DM) is used to overlap the probe beam and the alignment beam, followed by a plano-convex lens $(F L, f=30 \mathrm{~cm}$ ) which focuses the two collinear beams into the vacuum chamber via the entrance window (EW). The foci of the laser beams are positioned at the point where the laser paths cross the molecular beam. The focal spot size, $\omega_{0}$, of the alignment (probe) beam is $35 \mu \mathrm{m}(20 \mu \mathrm{m})$. The intensity of the alignment pulses is controlled by the combination of a half-wave plate (HWP) and a thin film polarizer (TFP). The alignment pulse polarization is controlled by passing it through a rotatable half-wave plate (HWP), followed by a quarter-wave plate (QWP) with its fast axis fixed in the horizontal plane.

In most of the measurements, the alignment pulse is linearly polarized corresponding to 1D alignment of the molecules. Photoelectron images were then recorded for the following three cases: i) no alignment pulse, i.e. randomly aligned molecules, ii) the polarizations of the alignment pulse and probe pulse parallel to each other, iii) the polarizations of the alignment pulse and probe pulse perpendicular to each other. In the case of naphthalene, measurements were also conducted on 3D aligned molecules, obtained by using an elliptically polarized alignment pulse.

Before travelling through the dichroic mirror (DM), the pulses in the alignment beam are spectrally truncated by a long-pass transmission filter (LPTF). This leads to a temporal truncation where the intensity drops by more than a factor of 100 over $\sim 10$ ps. ${ }^{33,34}$ The resulting intensity profile of the alignment pulse, obtained by cross correlation with the probe pulse, is shown in the inset of Fig. 1c. The timing of the probe pulse with respect to the alignment pulse is controlled by a delay stage (DS2) in the probe beam, placed after the KDP crystal.

The experimental procedure yields raw images that result from a 3D distribution of photoelectrons impacting on a 2D detector. Because cylindrical symmetry has been maintained in the experiment the 3D distribution can be recovered through Abel inversion and this was achieved using the BASEX software. ${ }^{35}$ Radial and angular distributions were extracted from the 3D distribution, with the radial distribution converted to a photoelectron spectrum (kinetic energy distribution) using the 
appropriate Jacobian and following calibration. The angular distribution for a given photoelectron peak was obtained by summing the intensity as a function of angle over the whole peak area. This distribution was then fit to a series of even Legendre polynomials, $P_{L}\left(\cos \theta_{\mathrm{LF}}\right)$, up to $L=10$, where "LF" denotes the laboratory frame.

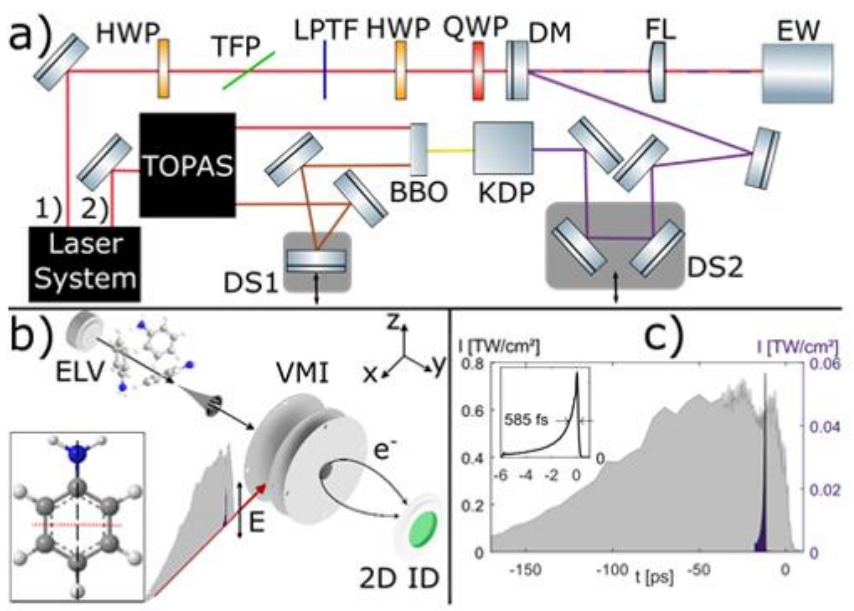

Fig. 1: (a) Schematic of the laser set-up showing the paths of the alignment beam (1) and the probe beam (2). HWP: Half-wave plate, QWP: Quarter-wave plate, TFP: Thin-film polarizer, LPTF: Long-pass transmission filter, DM: Dichroic mirror, FL: Focusing lens, EW: entrance window, DS: delay stage. The remaining non-labelled components are mirrors. (b) Schematic of the key elements of the experiment. The alignment and the probe pulse are both linearly polarized along the z-axis. ELV: Even-Lavie valve, 2D ID: 2D imaging detector, VMI: velocity map imaging electrodes. The inset shows a sketch of the molecular structure of aniline and the most polarizable axis (MPA, black dashed line) and its transition dipole moment (red dotted line). (c) The grey shaded area shows the intensity profile of the alignment pulse, measured via cross-correlation with the probe pulse. The probe pulse is indicated by the narrow violet peak and shown in the inset.

\section{Theory}


The various axes and angles that are referred to throughout this section and in the rest of this paper are defined in Fig. 2. Molecular frame photoelectron angular distributions (MFPADs) were generated using radial dipole matrix elements calculated by performing quantum scattering calculations using the ePolyScat package. ${ }^{36,37}$ In order to generate the initial and final state electronic wavefunctions for the scattering calculations the molecular orbitals were generated on geometry-optimized $\mathrm{S}_{0}$ structures at the HF/aug-cc-pVDZ level using the GAMESS software package. $^{38,39}$ The scattering calculations produced radial dipole matrix elements in the form shown in Eq. (1). ${ }^{36,37}$

$$
I_{l, m, \mu}^{p_{i} \mu_{i}, p_{f} \mu_{f}}(E)=\left\langle\Psi_{i}^{p_{i}, \mu_{i}}\left|\hat{d}_{\mu}\right| \Psi_{f}^{p_{f}, \mu_{f}} \varphi_{k l m}^{(-)}\right\rangle
$$

In Eq. (1) $I_{l, m, \mu}^{p_{i} \mu_{i}, p_{f} \mu_{f}}(E)$ is the radial dipole matrix element at the photoelectron kinetic energy, $E$, $\Psi_{i}^{p_{i}, \mu_{i}}$ and $\Psi_{f}^{p_{f}, \mu_{f}}$ are the initial and final electronic wavefunctions, $\hat{d}_{\mu}$ is the dipole operator and $\varphi_{k l m}^{(-)}$is the photoelectron wavefunction.
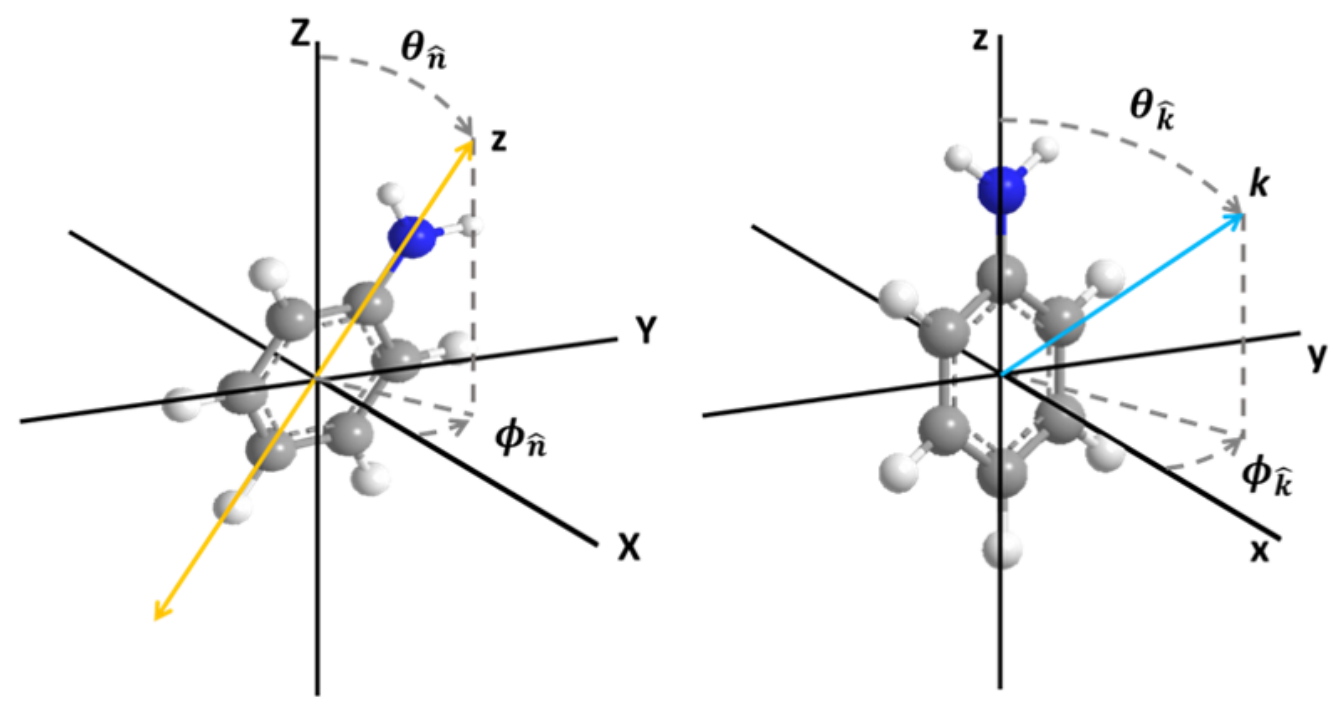

Fig. 2: Relationship between the laboratory frame $(X, Y, Z)$ and molecular frame $(x, y, z)$ axis systems and the emission direction of the photoelectron for aniline. The most polarisable molecular axis (MPA) is shown in yellow in the left-hand panel and defined as $z$. The direction of photoelectron ejection is shown in blue and defined as k. $\left(\theta_{\hat{n}}, \phi_{\hat{n}}\right)$ defines the orientation of the MPA with respect to the laboratory frame. The 
photoelectron emission direction is given by $\left(\theta_{\hat{k}}, \phi_{\hat{k}}\right)$ in the molecular frame and by $\left(\theta_{L F}, \phi_{L F}\right)$ in the laboratory frame.

The radial dipole matrix elements were rotated into the frame needed to define a specific geometry of interaction between the molecule and electric field according to Eq. (2);

$$
T_{\mu_{0}}^{p_{i} \mu_{i}, p_{f} \mu_{f}}\left(\theta_{\hat{k}}, \phi_{\hat{k}}, \theta_{\hat{n}}, \phi_{\hat{n}}\right)=\sum_{l, m, \mu} I_{l, m, \mu}^{p_{i} \mu_{i}, p_{f} \mu_{f}}(E) Y_{l m}^{*}\left(\theta_{\hat{k}}, \phi_{\hat{k}}\right) D_{\mu,-\mu_{0}}^{1}\left(R_{\hat{n}}\right)
$$

Here, $T_{\mu_{0}}^{p_{i} \mu_{i}, p_{f} \mu_{f}}\left(\theta_{\hat{k}}, \phi_{\hat{k}}, \theta_{\hat{n}}, \phi_{\hat{n}}\right)$ is the resulting matrix element, $\mathrm{Y}_{l m}^{*}\left(\theta_{\hat{k}}, \phi_{\hat{k}}\right)$ is a spherical harmonic function and $D_{\mu,-\mu_{0}}^{1}\left(R_{\hat{n}}\right)$ is the Wigner rotation matrix. The emission direction of the photoelectron is denoted by $\left(\theta_{\hat{k}}, \phi_{\hat{k}}\right)$, whereas $\left(\theta_{\hat{n}}, \phi_{\hat{n}}\right)$ denotes the angle between the polarization vector of the probe pulse and the highest symmetry axis, which in this case is also the most polarizable axis (MPA). In the case of aniline this is the principal molecular axis indicated by the black dashed line in Fig. 1b. The MFPADs are determined by the square of these matrix elements, $I_{\mu_{0}}^{p_{i}, p_{f}}\left(\theta_{\hat{k}}, \phi_{\hat{k}}, \theta_{\hat{n}}, \phi_{\hat{n}}\right)$, according to Eq. 3.

$$
I_{\mu_{0}}^{p_{i}, p_{f}}\left(\theta_{\hat{k}}, \phi_{\hat{k}}, \theta_{\hat{n}}, \phi_{\hat{n}}\right)=\frac{4 \pi^{2} E}{c g_{p_{i}}} \sum_{\mu_{i}, \mu_{f}}\left|T_{\mu_{0}}^{p_{i} \mu_{i}, p_{f} \mu_{f}}\left(\theta_{\hat{k}}, \phi_{\hat{k}}, \theta_{\hat{n}}, \phi_{\hat{n}}\right)\right|^{2}
$$

In the case of aniline, the initial wavefunction was taken to be the molecular orbital with the largest contribution to the $S_{1}$ excited state ${ }^{40}$ and the final ionic state populated is the initial state minus a single electron. The naphthalene calculations are complicated by the fact that the excited state has mixed electronic character ${ }^{41}$. Here we have performed the calculations for the $S_{2}$ component, which in turn we have represented as arising from a single excitation which in fact contributes only $76 \% .{ }^{42}$ Details of the method can be found in Ref. 43. The continuum wavefunction, and hence the MFPAD, was then calculated at a series of photoelectron kinetic energies and angles $\left(\theta_{\hat{n}}, \phi_{\hat{n}}\right)$. Full computational results can be found online, via the ePSdata project. ${ }^{44,45}$ 
The MFPAD depends on the photoelectron kinetic energy, $E$, and on $\left(\theta_{\hat{n}}, \phi_{\hat{n}}\right)$, (see Fig. 2); because of the 3D structure of the molecules the MFPAD will not be cylindrically symmetric. Therefore, the MFPAD can be expressed as: ${ }^{46}$

$$
I^{M F}\left(\theta_{\hat{k}}, \phi_{\hat{k}} ; E, \theta_{\hat{n}}, \phi_{\hat{n}}\right) \propto \sum_{L M} \mathrm{~B}_{L M}^{M F} Y_{L M}\left(\theta_{\hat{k}}, \phi_{\hat{k}}\right)
$$

where the number of terms depends only on the maximum orbital angular momentum, $I_{\max }$ carried away by the ejected photoelectron; $L_{\max }=2 I_{\max }$.

The ePolyScat calculations also provide values of $\beta$, the coefficient of $P_{2}\left(\cos \theta_{L F}\right)$ in the photoelectron angular distribution that arises following ionization of an isotropic distribution of MPAs. In the case of aniline, the trend in these values as a function of photoelectron kinetic energy can be compared with the trend in the values of the equivalent coefficient that have been determined in $(1+1)$ two-photon ionization experiments by Qu et al. ${ }^{47}$ and by Thompson et al. ${ }^{48}$ Although the experimental values will be influenced by the small anisotropy introduced in the twophoton ionization process, in both cases the coefficient of $P_{2}\left(\cos \theta_{L F}\right)$ decreases from a positive value at $\sim 0 \mathrm{eV}$ to zero at $\sim 0.5 \mathrm{eV}$ and is then increasingly negative from $\sim 0.5 \mathrm{eV}$ to $\sim 1 \mathrm{eV}$. This comparison therefore enables us to verify the computational results and that the calculated energies are in good agreement with the experimental energies.

Two separate processes were used to generate the simulated photoelectron images, one for ionization of a "completely aligned" sample $\left(\left\langle\cos ^{2} \theta_{\hat{n}}\right\rangle=1\right.$ or $\left.\left\langle\cos ^{2} \theta_{\hat{n}}\right\rangle=0\right)$ and one for ionization of an isotropic sample $\left(\left\langle\cos ^{2} \theta_{\hat{n}}\right\rangle=1 / 3\right)$. For the completely aligned sample, a full 3D velocity distribution for a single component of the ionisation continuum $\left(a_{u} / b_{1 u}\right.$ for naphthalene; $a_{2} / b_{1}$ for aniline) was created in the molecular frame using the radial dipole matrix elements at the chosen photoelectron kinetic energy. The molecular frame PAD was then rotated so that the most polarisable axis was aligned vertically $\left(a_{u} / a_{2}\right)$ or horizontally $\left(b_{1 u} / b_{1}\right)$ and then azimuthally averaged. The resulting 3D distribution was then projected onto a 2D plane to create simulated photoelectron images. For the isotropic sample a 3D laboratory frame PAD was created using the $\beta$ parameter 
calculated by ePolyScat at the chosen photoelectron kinetic energy and then projected onto a 2D plane to create simulated photoelectron images.

\section{Results}

\subsection{Naphthalene}

Naphthalene was ionized in a (1+1) scheme through the $\overline{8}^{1} \overline{7}^{1} 8^{1}$ vibrational state ${ }^{49}$ at $2052 \mathrm{~cm}^{-1}$ above the $S_{1}$ origin using $293.53 \mathrm{~nm}$ light linearly polarized along the $Z$ direction (Fig. 3). The raw photoelectron image that is measured in the absence of the alignment pulse is shown in Fig. 3a(ii). Two prominent rings are observed in the image, with a slight preference for photoelectron emission direction along the polarization of the probe polarization vector in both rings. The effect of introducing an alignment pulse that is linearly polarized along $Z$ can be seen in Fig. 3b(ii); this pulse is expected to induce 1D alignment, confining the long axis of the molecules along the $Z$ as illustrated in Fig. $3 b$ (i). In Fig. 3b(ii) it can be seen that, in the case of the outer ring, the intensity of the electron emission along the Z-axis decreases and a four-lobed structure appears. Measurements were also made with an elliptically polarized alignment beam to induce 3D alignment; in this case the major polarization axis was directed along the Z-axis and the minor polarization axis along the $\mathrm{Y}$-axis with an ellipticity ratio of 3:1. The resulting alignment is illustrated schematically in Fig. 3c(i). The outermost ring in the image shows the same characteristic four-lobed structure, see Fig. $3 c(i i)$, with the suppression of the photoelectron emission along the Z-axis a little more pronounced than when the molecules are 1D aligned.

Because cylindrical symmetry has been preserved to measure the images shown in Figs. $3 a$ and 3b the BASEX software can be used to invert the images. However, when the naphthalene molecules are aligned in three dimensions (Fig. 3c) cylindrical symmetry is broken. Although alternative routines, such as FINA, ${ }^{50,51}$ do exist to deal with this situation, in this work we have not determined a 
kinetic energy distribution or angular distribution for the image shown in Fig. $3 \mathrm{c}$ so this image will not be discussed in what follows.

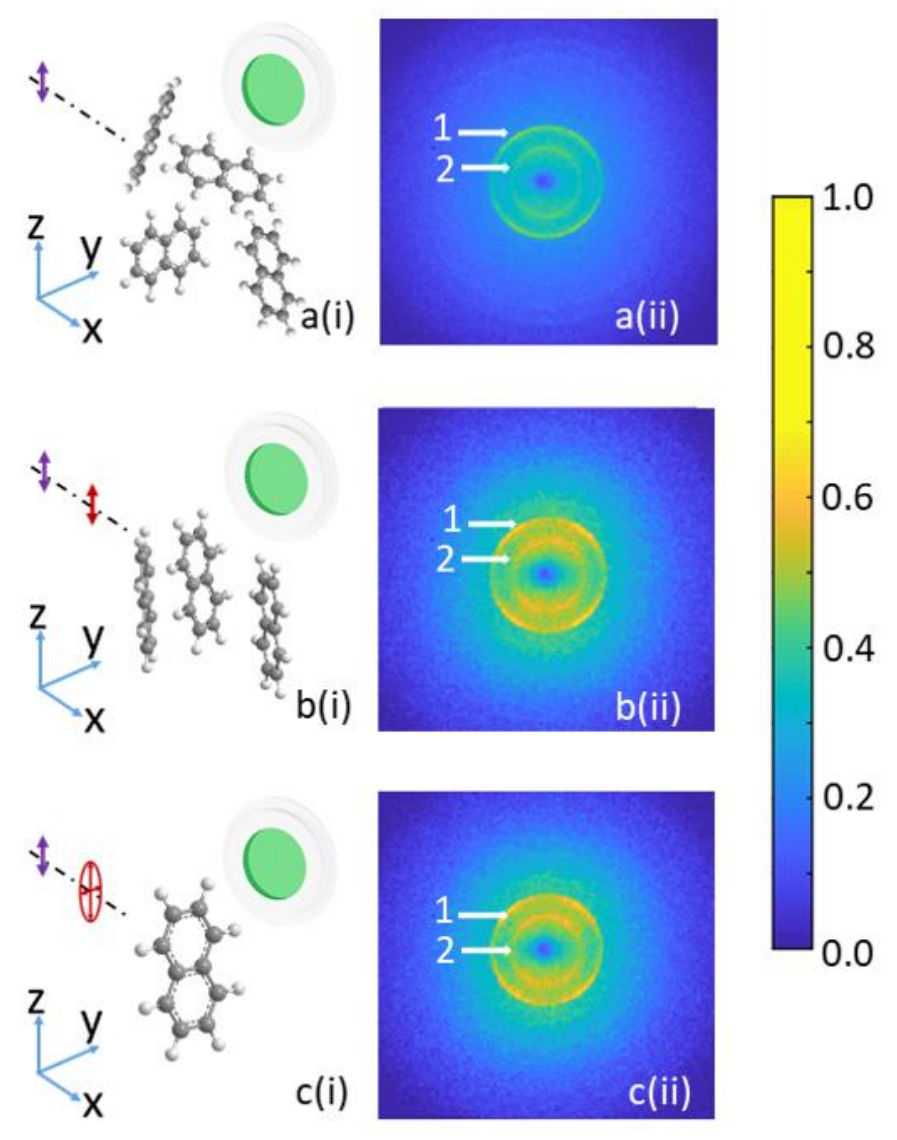

Fig. 3 Photoelectron images from naphthalene; the intensity in these images has been scaled by the radius for presentational purposes. The purple arrow indicates the direction of the probe polarisation and the red arrow indicates the direction of the alignment polarisation. (a) No alignment pulse present (isotropic sample), (i) spatial orientations of the naphthalene molecules that are ionized relative to the detector; (ii) raw photoelectron image produced when the $293.53 \mathrm{~nm}$ probe pulse ionizes the naphthalene molecules. (b) as for (a) but with the linearly polarized alignment pulse introduced. (c) as for (a) but with the elliptically polarized alignment pulse introduced, with an intensity ratio between the major and minor axis of 3:1. The peak intensity of the alignment pulse is $5.8 \times 10^{11} \mathrm{~W} / \mathrm{cm}^{2}$. 
The rings observed in the image appear as distinct peaks in the kinetic energy distribution, shown for randomly oriented molecules by the blue curve in Fig. 4 . The peak corresponding to the outermost ring is labelled peak 1 in Fig. 4 and centered at $0.33 \mathrm{eV}$. This peak corresponds to the formation of the naphthalene cation in its vibrational ground state. Peak 2 corresponds to the next ring and is centered at $0.12 \mathrm{eV}$; this peak corresponds to the formation of the naphthalene cation in an excited vibrational state that is likely to be the $\overline{8}^{1} 7^{1}$ state that was observed at an ion energy of $1503 \mathrm{~cm}^{-1}$ (photoelectron kinetic energy of $953 \mathrm{~cm}^{-1}$ ) by Cockett et al..$^{52}$ following ionization of the $\overline{8}^{1} 8^{1}$ state at $1135 \mathrm{~cm}^{-1}$ above the $S_{1}$ origin. The kinetic energy distribution extends almost to $2 \mathrm{eV}$, even with no alignment pulse present. Such high energies indicate that the naphthalene molecule can absorb three photons from the probe pulse. The kinetic energy distribution that results when the molecules are aligned in one dimension by the $800 \mathrm{~nm}$ pulse is shown by the red curve in Fig. 4. It can be seen that the alignment pulse only causes minor changes in the photoelectron spectrum.

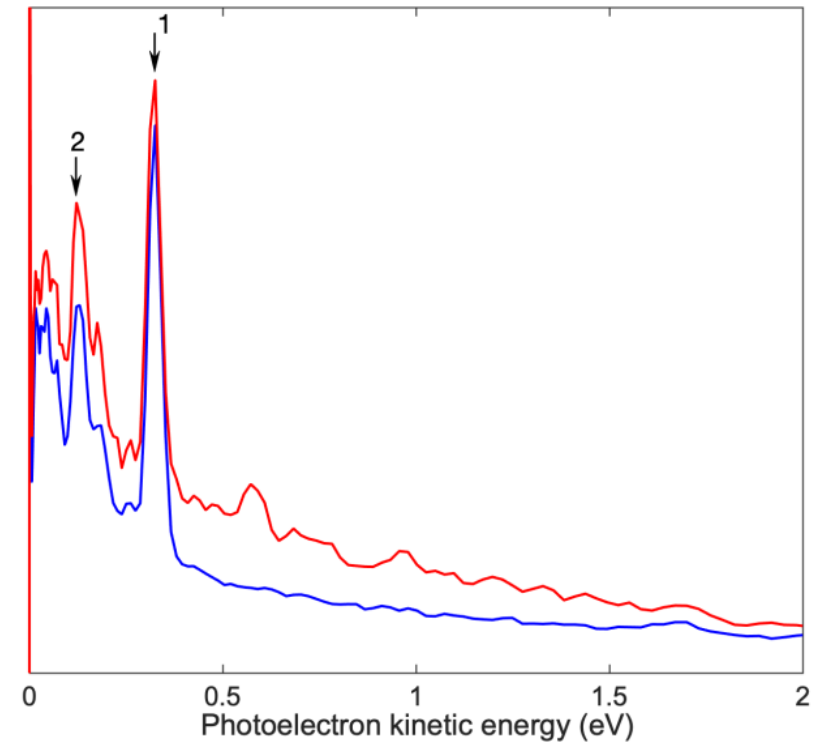

Fig. 4 Kinetic energy distribution of the photoelectrons produced when the $293.53 \mathrm{~nm}$ probe pulse ionizes naphthalene molecules. The blue curve corresponds to randomly oriented molecules and is obtained from the image in Fig. 3a(ii). The red curve corresponds to 1D aligned molecules and is obtained from the image in Fig. 3b(ii). The baseline corresponds to zero intensity. 
Angle-resolved photoelectron intensities, $I^{L F}\left(\theta_{L F}\right)$, are obtained for the main photoelectron peaks, where $\theta_{L F}$ is the polar angle between the electron emission direction in the detector plane and the laboratory frame (LF) axis defined by the polarization of the probe beam. The angular distributions can be quantified by fitting $I^{L F}\left(\theta_{L F}\right)$ to an expansion in Legendre polynomials:

$$
I^{L F}\left(\theta_{L F}\right) \propto 1+\mathrm{b}_{2}^{L F} P_{2}\left(\cos \theta_{L F}\right)+\mathrm{b}_{4}^{L F} P_{4}\left(\cos \theta_{L F}\right) \ldots \mathrm{b}_{L}^{L F} P_{L}\left(\cos \theta_{L F}\right)
$$

to obtain values of the coefficients $\mathrm{b}_{L}^{L F}$. In Eq. (5) $\mathrm{b}_{L}^{L F}$ is a normalised laboratory frame anisotropy parameter and $P_{L}\left(\cos \theta_{L F}\right)$ is a Legendre polynomial, both of degree $L$. In general, the number of terms required in the expansion depends on the experimental geometry, the number of photons, the degree of alignment, and the maximum orbital angular momentum associated with the departing photoelectron. Because the experiment has cylindrical symmetry and inversion symmetry there is no dependence on the azimuthal angle $\phi_{L F}$ and only even values of $L$ contribute to the series. In practice, we find that the inclusion of terms with $L>10$ does not improve the fit.

Fitting the photoelectron intensity (determined through reconstruction) as a function of angle enables $\mathrm{b}_{L}^{L F}$ parameters to be deduced for both rings in the images shown in Figs. 3a and 3b; these are shown in Table 1. The four-lobed structure associated with peak 1 when the alignment pulse is present means that the angular distribution must contain a significant non-zero contribution from $\mathrm{b}_{L}^{L F}$ coefficients with $L \geq 4$. Inspection of Table 1 shows that the magnitude of $\mathrm{b}_{4}^{L F}$ is significant for both of the photoelectron peaks analysed in naphthalene, but that in the case of peak $1\left|b_{4}^{L F}\right| \gg$ $\left|b_{2}^{L F}\right|$, consistent with the characteristic four-lobed shape observed. 
Table 1: Experimental anisotropy parameters, $\mathrm{b}_{L}^{L F}$, that describe the photoelectron angular distributions observed for naphthalene. The approximate error bar is \pm 0.01 .

\begin{tabular}{|c|c|c|c|c|}
\hline \multirow{2}{*}{$\mathrm{b}_{L}^{L F}$} & \multicolumn{2}{|c|}{$\begin{array}{c}\text { Photoelectron intensity over the } \\
\text { range 0.30-0.36 eV } \\
\text { (peak 1) }\end{array}$} & $\begin{array}{c}\text { Photoelectron intensity over the } \\
\text { range 0.09-0.15 eV } \\
\text { (peak 2) }\end{array}$ \\
\hline & unaligned & 1D aligned & unaligned & 1D aligned \\
\hline $\mathrm{b}_{2}^{L F}$ & 0.28 & 0.25 & 0.30 & 0.23 \\
\hline $\mathrm{b}_{4}^{L F}$ & -0.20 & -0.46 & -0.15 & -0.30 \\
\hline $\mathrm{b}_{6}^{L F}$ & - & -0.06 & - & -0.07 \\
\hline $\mathrm{b}_{8}^{L F}$ & - & 0.04 & - & 0.09 \\
\hline $\mathrm{b}_{10}^{L F}$ & - & 0.06 & - & -0.08 \\
\hline
\end{tabular}

In order to gain insight into the measured photoelectron anisotropy we have performed ePolyScat calculations of the photoionization dynamics in order to determine MFPADs, as described in Section 3. In Fig. 5 we show examples of the calculated MFPADs at different photoelectron kinetic energies and in two different geometries: $\left(\theta_{\hat{n}}, \phi_{\hat{n}}\right)=(0,0)$ and $\left(\theta_{\hat{n}}, \phi_{\hat{n}}\right)=\left(\frac{\pi}{2}, \frac{\pi}{2}\right)$. These geometries select photoelectron partial waves of $a_{u}$ and $b_{1 u}$ symmetry, respectively. The dramatic change in the MFPADs over the $0.13-4.0 \mathrm{eV}$ range of kinetic energy can be attributed to the presence of a shape resonance by analogy with experimental observations on other similar molecules. ${ }^{53,54}$ 
$0.13 \mathrm{eV}$

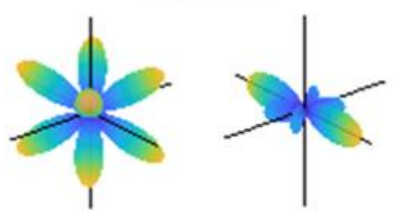

$2.00 \mathrm{eV}$
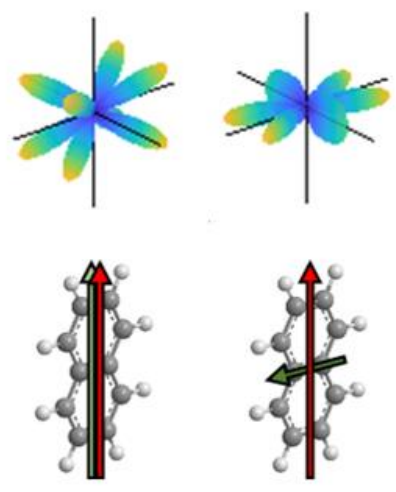

$0.33 \mathrm{eV}$

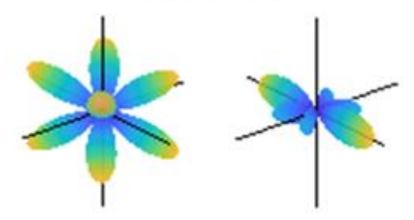

$3.00 \mathrm{eV}$
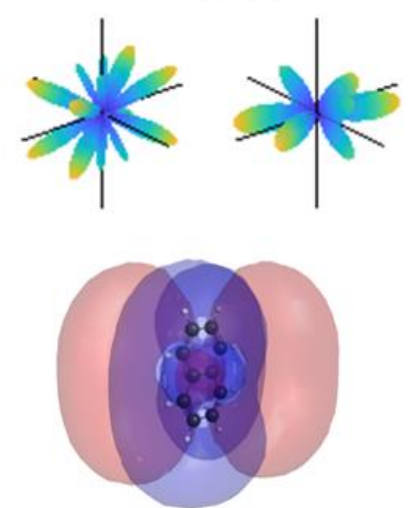

$1.00 \mathrm{eV}$

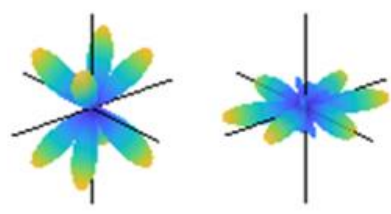

$4.00 \mathrm{eV}$

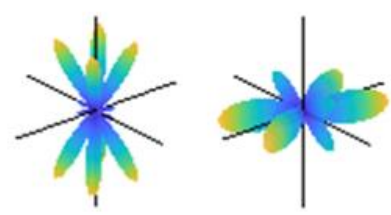

Fig. 5: Spherical polar plots showing MFPADs following the ionization of naphthalene at different photoelectron kinetic energies. At each kinetic energy two MFPADs are shown; one for the case where the polarization vector of the ionizing beam (green) is parallel to the MPA (red); this selects the $a_{u}$ continuum, and one for the case where the polarization vector of the ionizing beam is perpendicular to the MPA; this selects the $b_{1 u}$ continuum. The molecular orbital used to describe the initial electronic state of naphthalene is shown on the bottom right.

The MFPADs are relatively simple and have up-down symmetry at all kinetic energies. As the kinetic energy increases, more lobes appear in the MFPADs for both polarization geometries. The LFPAD results from an average over all the possible directions of the MPA in the laboratory frame, weighted by any anisotropy in the distribution of MPAs that is introduced by the experiment by the alignment pulse. When the alignment pulse is introduced the distribution of MPAs is confined along the laboratory frame $Z$ axis, according to the value of $\left\langle\cos ^{2} \theta_{\hat{n}}\right\rangle$. In the limit of $\left\langle\cos ^{2} \theta_{\hat{n}}\right\rangle=1$ or $\left\langle\cos ^{2} \theta_{\hat{n}}\right\rangle=0$ the LFPAD would converge on the $a_{u}$ or $b_{1 u}$ MFPAD, respectively. In order to establish how close to a molecular frame measurement we have achieved in the photoelectron images shown in Figs. 3b, we have used the output of the ePolyScat calculations at $0.33 \mathrm{eV}$ photoelectron kinetic 
energy to create simulated photoelectron images in three limiting cases: (a) ionization of an isotropic distribution of naphthalene molecules $\left(\left\langle\cos ^{2} \theta_{\hat{n}}\right\rangle=1 / 3\right)$, (b) ionization of a distribution of naphthalene molecules whose MPAs are completely aligned along the polarization vector of the ionizing beam ( $\left\langle\cos ^{2} \theta_{\hat{n}}\right\rangle=1$; this selects the $a_{u}$ continuum), and (c) ionization of a distribution of naphthalene molecules whose MPAs are completely aligned perpendicular to the polarization vector of the ionizing beam $\left(\left\langle\cos ^{2} \theta_{\hat{n}}\right\rangle=0\right.$; this selects the $b_{1 u}$ continuum). The results of these simulations are shown in Fig. 6 and can be compared with the equivalent experimental images; we have also included in Fig. 6 the experimental photoelectron image that results when the polarization of the alignment beam is perpendicular to the polarization of the probe beam.

When an isotropic sample is ionized the photoelectron wave function will contain contributions from all of the possible ionization continua which are defined by the photoelectron orbital angular momentum, $l$, and the irreducible representation $\chi$. In the case of naphthalene, the possible continua are $l a_{u}, l b_{1 u}$, and $l b_{3 u}$. The PAD is determined by the relative magnitude of each component (dictated by the radial dipole matrix element) and the relative phase between components. Because the phase contribution is complicated, and controls the extent of constructive and destructive interference, it is not possible to simply predict what the laboratory frame PAD is expected to look like on the basis of the plots shown in Fig. 5. However, the ePolyScat code determines $\beta$, the coefficient of $P_{2}\left(\cos \theta_{L F}\right)$ in the photoelectron angular distribution that arises following ionization of an isotropic distribution of MPAs; this value is used to generate the image shown in Fig. $6 \mathrm{~d}$. In the experiment, a resonant two-photon ionization scheme is used and so the sample ionized is not fully isotropic. Nonetheless, the photoelectron intensity peaks along the vertical axis in Fig. 6a, consistent with the simulation shown in Fig. $6 \mathrm{~d}$. 

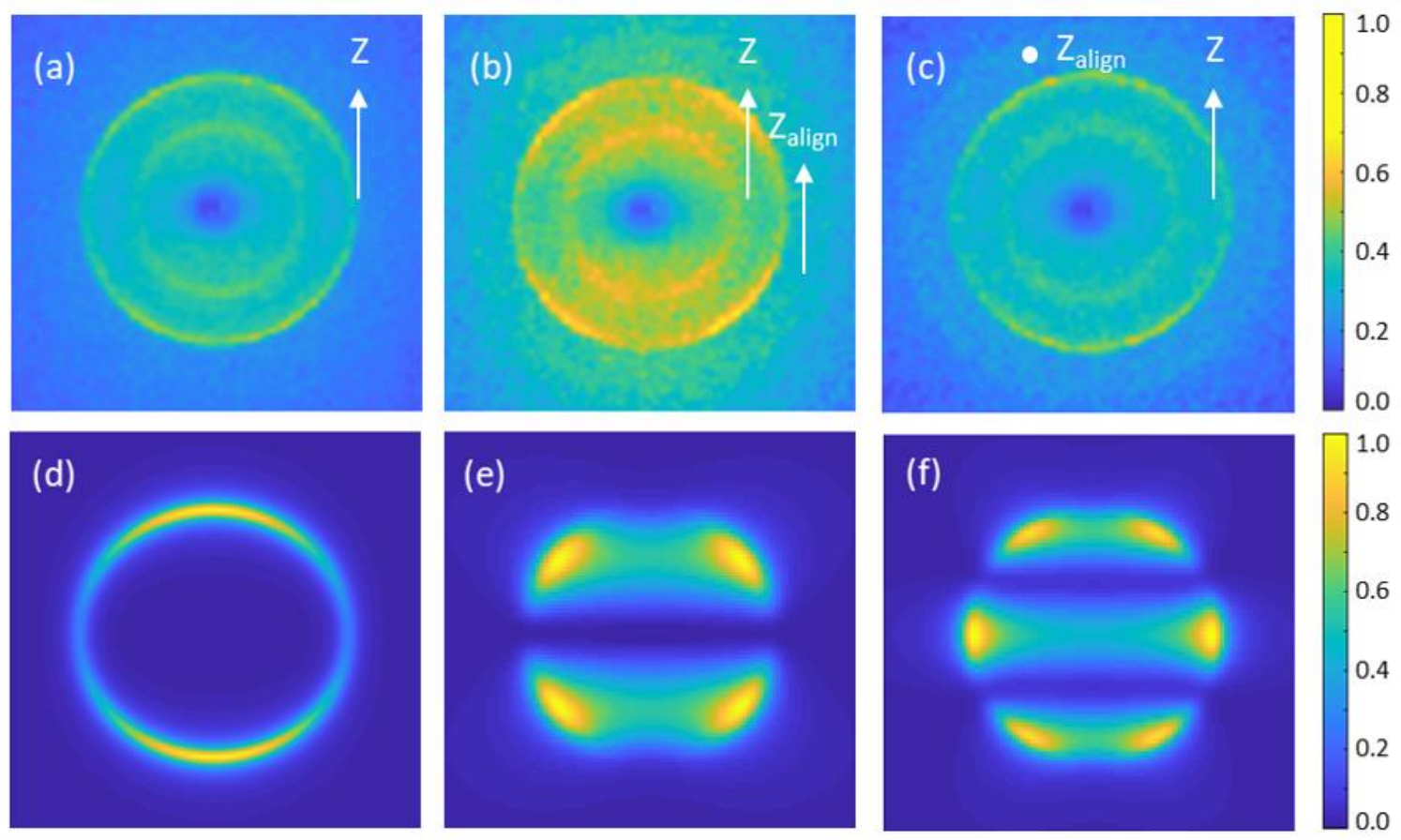

Fig. 6 Comparison of experimental and simulated photoelectron images of naphthalene, where the intensity in the experimental images has been scaled by the radius for presentational purposes. In all cases the polarization vector of the ionizing beam, $Z$, points vertically. Panels (a)-(c) show the raw experimental images for (a) probe only (isotropic sample), (b) alignment and probe polarizations parallel (taken from Fig. 3b) and (c) alignment and probe polarizations perpendicular. Panels (d)-(f) show shows the simulated photoelectron images for $0.33 \mathrm{eV}$ photoelectron kinetic energy for (d) an unaligned sample, (e) a sample with $\left\langle\cos ^{2} \theta_{\hat{n}}\right\rangle=1$ ( $a_{u}$ continuum) and (f) $\left\langle\cos ^{2} \theta_{\hat{n}}\right\rangle=0\left(b_{1 u}\right.$ continuum). The radius of the ring in the simulated images is chosen arbitrarily, and the experimental images have been scaled to match.

When a fully aligned sample is ionized a single irreducible representation is selected; $l a_{u}$ when the ionization beam is polarized along the MPA and, in our experimental setup, $l b_{1 u}$ when it is polarized perpendicular to the MPA. In the experiment we cannot achieve complete alignment, but as the alignment improves we expect to converge on the $l a_{u}$ result in the parallel geometry and the $l b_{1 u}$ result in the perpendicular geometry. In Fig. 6e we can see that the four-fold structure that appears in the outer ring of the experimental images when the alignment beam is introduced in the parallel geometry is reproduced in the simulations, suggesting that a high degree of alignment must have 
been achieved and that the measured PADs from naphthalene are close to the molecular frame result. Furthermore, the simulations also broadly reproduce the experimental image in the crossed polarization case. This agreement is remarkable considering that (i) the complicated nature of the excited electronic state in naphthalene has not been fully accounted for in the calculations, and (ii) the experimental alignment is not perfect.

\subsection{Aniline}

A distribution of randomly oriented aniline molecules [Fig. 7a(i)] was excited to the $S_{1}$ origin using light at $293.85 \mathrm{~nm}$ that is linearly polarized along $Z$ and ionized following the absorption of a second photon from the same laser beam. The resulting raw photoelectron image is shown in Fig. 7a(ii) and shows three prominent rings, labelled 1-3, with the photoelectron intensity approximately isotropic in all rings. Fig. $7 b$ shows the effect that introducing an alignment pulse, linearly polarized along $Z$, has on the observed photoelectron image. This pulse causes the molecules to align such that the distribution of MPAs is confined with respect to the polarization direction of the probe pulse, as indicated in Fig. $7 \mathrm{~b}(\mathrm{i})$. The raw image is shown in Fig. $7 \mathrm{~b}$ (ii) and can be compared with the image in Fig. 7a(ii) which was obtained without the alignment pulse. Two prominent changes can be seen: first, the rings are now clearly anisotropic with an enhanced intensity along the z-axis, and secondly, evidence of additional rings can be seen. 


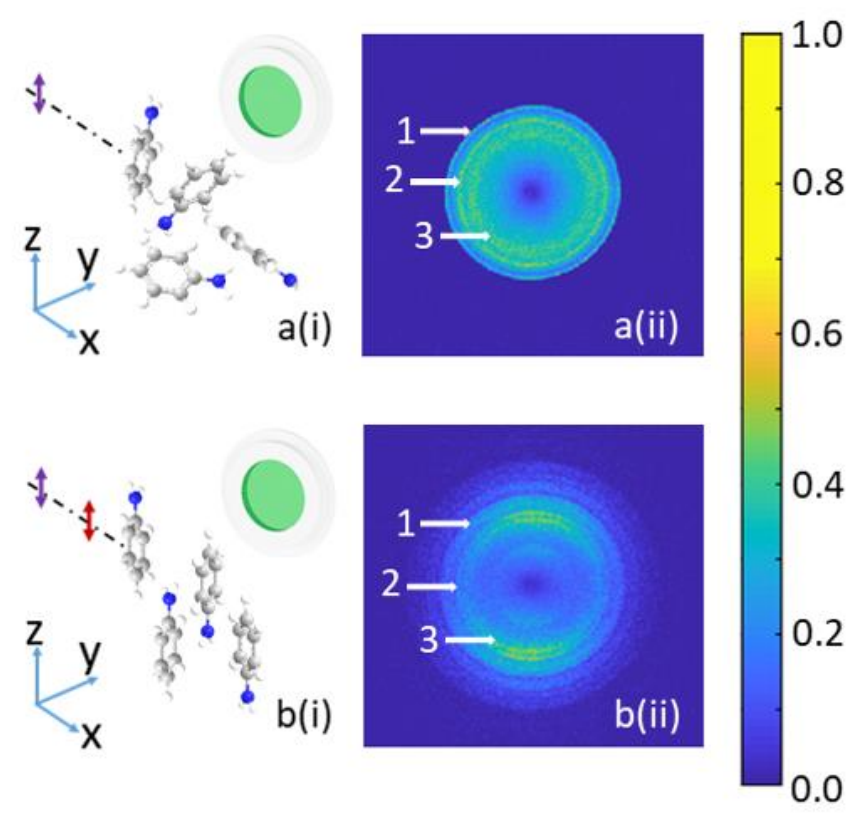

Fig. 7:

Photoelectron images from aniline; the intensity in these images has been scaled by the radius for presentational purposes. The purple arrow indicates the direction of the probe polarisation and the red arrow indicates the direction of the alignment polarisation. (a) No alignment pulse present (isotropic sample), (i) spatial orientations of the aniline molecules that are ionized relative to the detector; (ii) raw photoelectron image produced when the $293.85 \mathrm{~nm}$ probe pulse ionizes the aniline molecules. (b) as for (a) but with the linearly polarized alignment pulse introduced. The peak intensity of the alignment pulse is $8.1 \times 10^{11} \mathrm{~W} / \mathrm{cm}^{2}$.

The resulting photoelectron spectra are shown in Fig. 8. The spectrum arising from the unaligned sample is consistent with the spectrum obtained by Qu and coworkers, ${ }^{47}$ considering the differences in experimental conditions. In this figure it can be seen that the peak with maximum intensity is centered at $0.75 \mathrm{eV}$, in agreement with our expectation for the kinetic energy of photoelectrons associated with the formation of the cation in its vibrational origin; ${ }^{47}$ the results discussed in this paper focus on these photoelectrons. The peaks at lower kinetic energies correspond to the formation of the cation in vibrationally excited states. The alignment pulse causes a significant effect on the photoelectron spectrum, as can be seen by comparing the red and blue lines in Fig. 8 . 
In particular, photoelectrons with kinetic energies up to $1.6 \mathrm{eV}$ are detected; higher than energetically possible following the absorption of two probe photons. This can be explained if some of the electronically excited aniline molecules absorb an $800 \mathrm{~nm}$ photon from the alignment pulse before absorbing the second UV photon in a $\left(1+1^{\prime}+1\right)$ ionization scenario. If this occurs, then aniline molecules that are ionized to create cations in the ground vibrational state would release photoelectrons with a kinetic energy of $\sim 2.27 \mathrm{eV}$. As we will see, this process significantly affects the anisotropy observed in the photoelectron images.

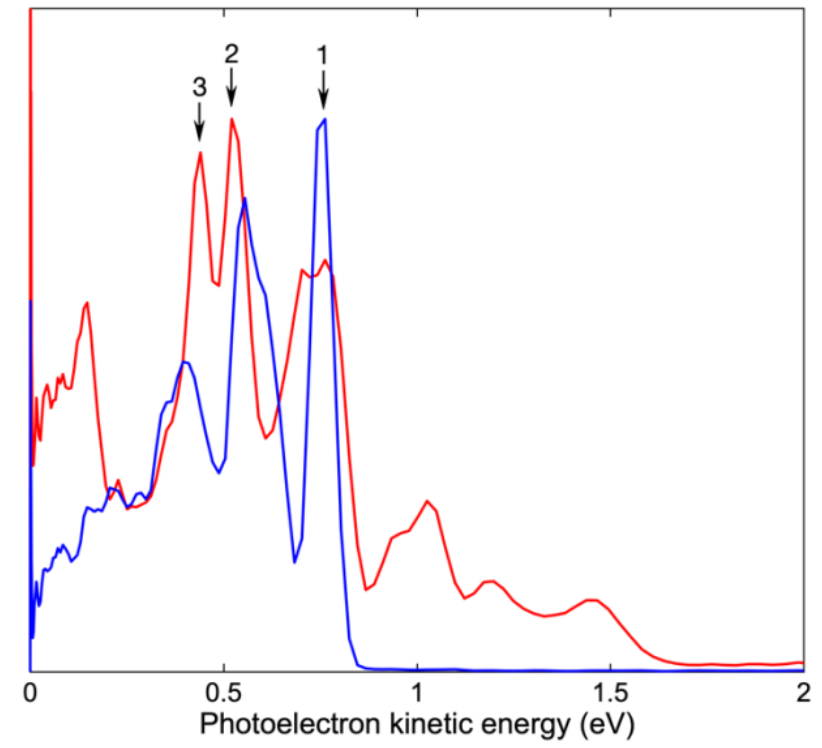

Fig. 8: Kinetic energy distribution of the photoelectrons produced when the $293.85 \mathrm{~nm}$ probe pulse ionizes aniline. The blue curve corresponds to randomly oriented molecules and is obtained from the image in Fig. 7a(ii). The red curve corresponds to $1 \mathrm{D}$ aligned molecules and is obtained from the image in Fig. $7 \mathrm{~b}(\mathrm{ii})$. The baseline corresponds to zero intensity.

In Fig. 9 we show examples of the resulting MFPADs at different photoelectron kinetic energies and in two different geometries: $\left(\theta_{\hat{n}}, \phi_{\hat{n}}\right)=(0,0)$ and $\left(\theta_{\hat{n}}, \phi_{\hat{n}}\right)=\left(\frac{\pi}{2}, \frac{\pi}{2}\right)$. These geometries select photoelectron partial waves of $a_{2}$ and $b_{1}$ symmetry, respectively. The MFPADs change dramatically 
over the $0.1-4.1 \mathrm{eV}$ range of kinetic energy, as expected from the experimental results presented by Qu et al. ${ }^{47}$

\section{$0.10 \mathrm{eV}$}

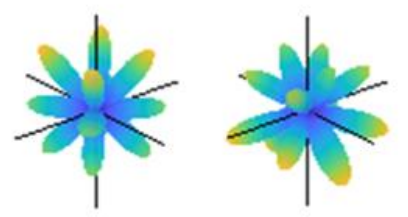

$2.10 \mathrm{eV}$
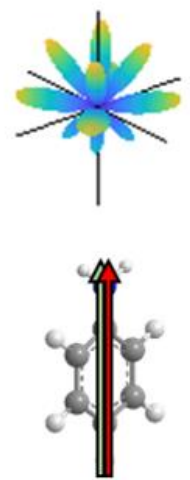

$0.7 \mathrm{eV}$

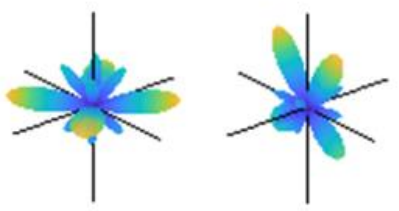

$3.10 \mathrm{eV}$
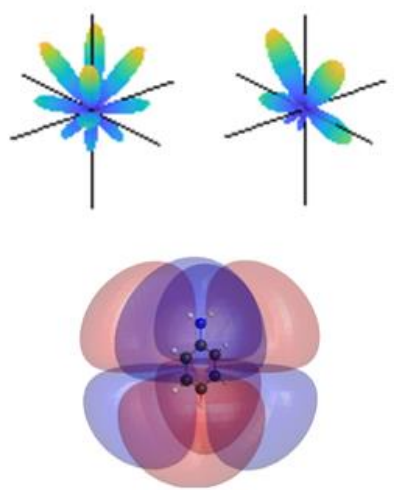

$1.10 \mathrm{eV}$

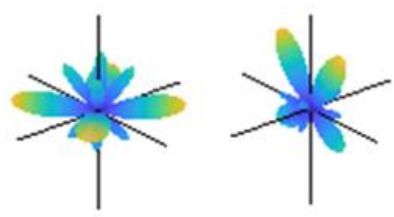

$4.10 \mathrm{eV}$

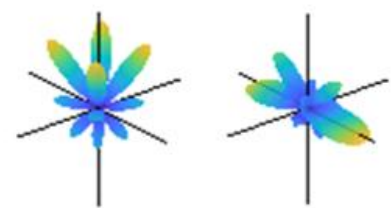

Fig. 9: Spherical polar plots showing MFPADs following the ionization of aniline at different photoelectron kinetic energies. At each kinetic energy two MFPADs are shown; one for the case where the polarization vector of the ionizing beam (green) is parallel to the MPA (red); this selects the $a_{2}$ continuum, and one for the case where the polarization vector of the ionizing beam is perpendicular to the MPA; this selects the $b_{1}$ continuum. The molecular orbital used to describe the initial electronic state of aniline is shown on the bottom right.

As with naphthalene, we have used the output of the ePolyScat calculations, in this case at the experimental photoelectron kinetic energy $(0.7 \mathrm{eV})$ corresponding to the outer ring in Fig. $7 \mathrm{~b}$, to create simulated photoelectron images in three limiting cases; (a) ionization of an isotropic distribution of aniline molecules $\left(\left\langle\cos ^{2} \theta_{\hat{n}}\right\rangle=1 / 3\right)$, (b) ionization of a distribution of aniline molecules whose MPAs are completely aligned along the polarization vector of the ionizing beam ( $\left\langle\cos ^{2} \theta_{\hat{n}}\right\rangle=1$; this selects the $a_{2}$ continuum), and (c) ionization of a distribution of aniline molecules whose MPAs are completely aligned perpendicular to the polarization vector of the 
ionizing beam $\left(\left\langle\cos ^{2} \theta_{\hat{n}}\right\rangle=0\right.$; this selects the $b_{1}$ continuum). The results of these simulations are shown in Fig. 10 below the equivalent experimental images. We have also included in Fig. 10 the experimental photoelectron image that results when the polarization of the alignment beam is perpendicular to the polarization of the probe beam.
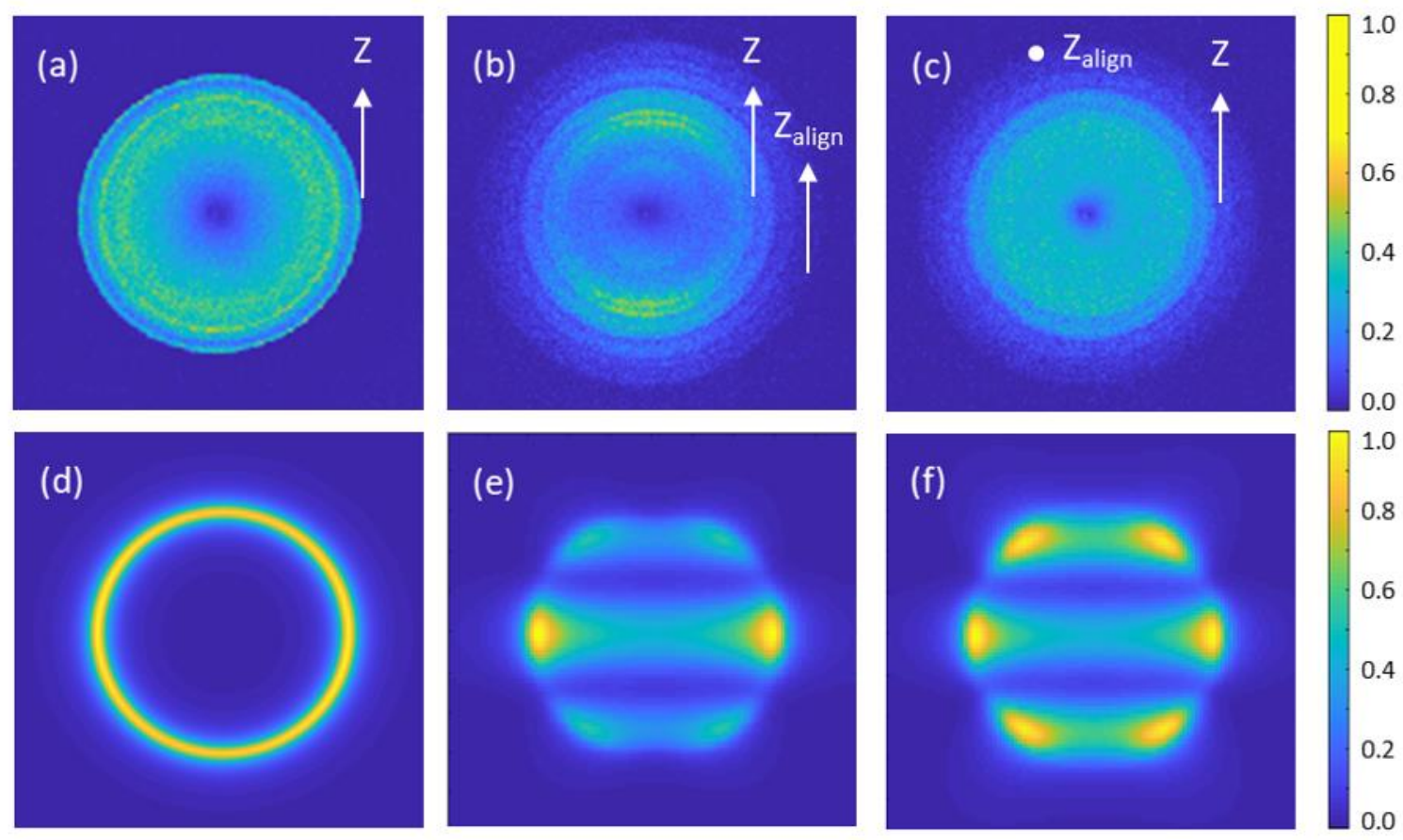

Fig. 10 Comparison of experimental and simulated photoelectron images of aniline; the intensity in the experimental images has been scaled by the radius for presentational purposes. In all cases the polarization vector of the ionizing beam, $Z$, points vertically. Panels (a)-(c) show the raw experimental images for (a) probe only (isotropic sample), (b) alignment and probe polarizations parallel (taken from Fig. 7b) and (c) alignment and probe polarizations perpendicular. Panels (d)-(f) show shows the simulated photoelectron images for $0.7 \mathrm{eV}$ photoelectron kinetic energy for (d) an unaligned sample, (e) a sample with $\left\langle\cos ^{2} \theta_{\hat{n}}\right\rangle=1\left(a_{2}\right.$ continuum) and (f) $\left\langle\cos ^{2} \theta_{\hat{n}}\right\rangle=0\left(b_{1}\right.$ continuum). The radius of the ring in the simulated images is chosen arbitrarily.

In Fig. 10 we can see that the simulated images do not reproduce what is seen in the experiment. In order to investigate whether this is caused by the fact that aniline does not align as well as 
naphthalene, a series of experimental photoelectron images was recorded at different intensities of the alignment pulse, with parallel polarizations of the alignment beam and the probe beam. In each case the angular distributions were fitted to Eq. (5) and the anisotropy parameters obtained from the fits are presented in Fig. 11. For the probe pulse only, the $\mathrm{b}_{L}^{L F}$ parameters are small with $\mathrm{b}_{2}^{L F}=$ -0.1 and $b_{4}^{L F}=0.06$, consistent with the near circularly-symmetric electron image. As the intensity of the alignment pulse is increased, the values of $b_{L}^{L F}$ with $L>2$ remain close to zero. Conversely, the value of $\mathrm{b}_{2}^{L F}$ increases steadily to a value of $\sim 0.4$, consistent with photoelectron intensity being concentrated along the direction of the polarization direction of the probe pulse. At higher alignment intensities, the value of $\mathrm{b}_{2}^{L F}$ continues to increase but at a much lower rate.

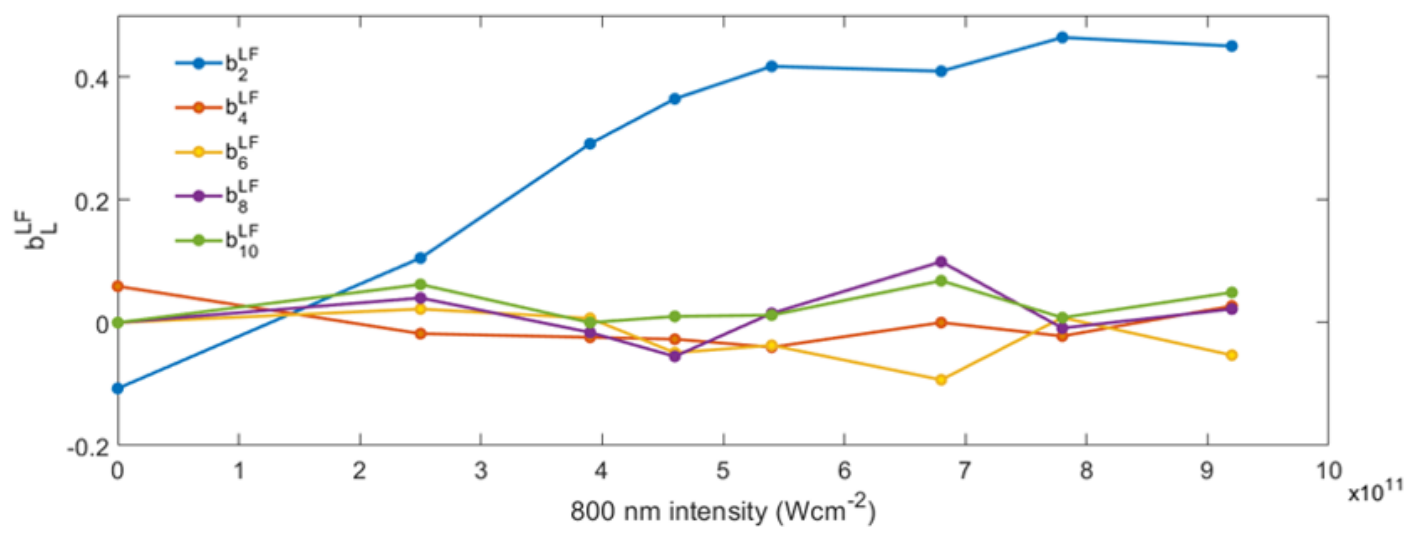

Fig. 11: Normalized photoelectron anisotropy parameters, $b_{\mathrm{L}}^{\mathrm{LF}}$, in the presence of different alignment field intensities, determined from the inversion of the measured aniline photoelectron images. All $b_{L}^{L F}$ values are assumed to contain an error of \pm 0.15 .

The change in the experimental values of $\mathrm{b}_{2}^{\mathrm{LF}}$ seen in Fig. 11 can be attributed to an increased degree of alignment, i.e. an increased value of $\left\langle\cos ^{2} \theta_{\hat{n}}\right\rangle$, as the intensity increases. ${ }^{55}$ The increasingly positive $\mathrm{b}_{2}^{\mathrm{LF}}$ values tell us that, as the alignment improves, the experimental photoelectron emission peaks increasingly parallel to the polarization vector of the probe beam. 
However, in Fig. $10 \mathrm{f}$ we have seen that the simulation for completely aligned aniline molecules shows photoelectron emission peaking perpendicular to the polarization vector of the probe beam. We can see that even for a high degree of alignment we do not expect the experimental images to converge on the molecular frame. We therefore attribute the discrepancy between the experimental and simulated images to the absorption of a $800 \mathrm{~nm}$ photon by aniline in its $\mathrm{S}_{1}$ electronic state. The absorption of the $800 \mathrm{~nm}$ photon will lead to excitation of a higher-lying electronic (Rydberg) state and allow access to higher-lying vibrational states in the cation. The evidence for this process can be seen in Fig. 8 where the photoelectron spectrum is qualitatively different when the alignment pulse is introduced.

\section{Conclusion}

We have presented photoelectron images measured following the ionization of aligned distributions of gas phase naphthalene and aniline molecules. The resulting images are found to exhibit anisotropy that increases when the alignment pulse is present with the aniline PADs peaking along the polarization vector of the ionizing light and the naphthalene PADs developing a characteristic four-lobed structure. Comparison with calculated photoelectron images that result from the ionization of unaligned and fully aligned distributions of molecules demonstrates that an excellent degree of alignment has been achieved in the case of naphthalene, and that the photoelectron images are largely determined by the photoelectron angular distribution in the molecular frame.

In many respects the aniline work represents a cautionary tale. It is clear that the presence of the alignment pulse provides an additional ionization channel involving the absorption of $800 \mathrm{~nm}$ photons by molecules that have been resonantly excited to $S_{1}$ by the UV pulse. Many other electronically excited molecules are likely to be similarly susceptible to the absorption of photons from an alignment pulse. We offer two ways to eliminate these unwanted processes. The first is to 
ionize molecules through absorption of a single VUV photon. In these cases, because no electronically excited states are involved, the most effective adiabatic alignment techniques can be used, allowing the measurement of PADs from complicated asymmetric top molecules that are close to fixed in space. However, studies of photophysical processes in aligned molecules require the preparation of electronically excited states. In these cases, perturbations caused by the alignment field can be avoided by turning it off once a sufficiently high degree of alignment has been achieved. Recently this technique has been demonstrated for a range of complex molecules embedded in helium nanodroplets. ${ }^{33}$ In these experiments a 150 ps long pulse, truncated to less than 10 ps at its peak, created sharp 1D or 3D alignment under field-free conditions lasting 10-20 ps. Although the field-free alignment will not last as long when created in gas phase molecules, it is expected to be sufficient to allow PADs from highly aligned molecules to be recorded. ${ }^{56}$

\section{Acknowledgements}

The research leading to these results has been enabled by funding from the Marie Skłodowska-Curie Actions of the European Union's Horizon 2020 framework under REA grant agreement no. 674960, and by EPSRC Grant EP/R010447. 


\section{References}

1. K. L. Reid, Molecular Physics 110, 131 (2012).

2. A. Yagishita, Journal of Electron Spectroscopy and Related Phenomena 200, 247 (2015).

3. P. Hockett, M. Staniforth, K. L. Reid and D. Townsend, Physical Review Letters 102, 253002 (2009).

4. E. Shigemasa, J. Adachi, M. Oura and A. Yagishita, Physical Review Letters 74, 359 (1995).

5. J. A. Davies, R. E. Continetti, D. W. Chandler and C. C. Hayden, Physical Review Letters 84, 5983 (2000).

6. M. S. Schöffler, J. Titze, N. Petridis, T. Jahnke, K. Cole, L. P. H. Schmidt, A. Czasch, D. Akoury, O. Jagutzki, J. B. Williams, N. A. Cherepkov, S. K. Semenov, C. W. McCurdy, T. N. Rescigno, C. L. Cocke, T. Osipov, S. Lee, M. H. Prior, A. Belkacem, A. L. Landers, H. Schmidt-Böcking, T. Weber and R. Dörner, Science 320, 920 (2008).

7. O. Geßner, A. M. D. Lee, J. P. Shaffer, H. Reisler, S. V. Levchenko, A. I. Krylov, J. G. Underwood, H. Shi, A. L. L. East, D. M. Wardlaw, E. t. H. Chrysostom, C. C. Hayden and A. Stolow, Science 311, 219 (2006).

8. T. Mizuno, J. Adachi, M. Kazama, M. Stener, P. Decleva and A. Yagishita, Physical Review Letters 110, 043001 (2013).

9. A. Menssen, C. S. Trevisan, M. S. Schöffler, T. Jahnke, I. Bocharova, F. Sturm, N. Gehrken, B. Gaire, H. Gassert, S. Zeller, J. Voigtsberger, A. Kuhlins, F. Trinter, A. Gatton, J. Sartor, D. Reedy, C. Nook, B. Berry, M. Zohrabi, A. Kalinin, I. Ben-Itzhak, A. Belkacem, R. Dörner, T. Weber, A. L. Landers, T. N. Rescigno, C. W. McCurdy and J. B. Williams, Journal of Physics B: Atomic, Molecular and Optical Physics 49, 055203 (2016).

10. H. Stapelfeldt and T. Seideman, Reviews of Modern Physics 75, 543 (2003).

11. K. L. Reid, Phil. Trans. R. Soc. A 376, 20170158 (2018).

12. V. Kumarappan, L. Holmegaard, C. Martiny, C. B. Madsen, T. K. Kjeldsen, S. S. Viftrup, L. B. Madsen and H. Stapelfeldt, Physical Review Letters 100, 093006 (2008). 
13. C. Z. Bisgaard, O. J. Clarkin, G. Wu, A. M. D. Lee, O. Gessner, C. C. Hayden and A. Stolow, Science 323, 1464 (2009).

14. P. Hockett, C. Z. Bisgaard, O. J. Clarkin and A. Stolow, Nat Phys 7, 612 (2011).

15. F. Kelkensberg, A. Rouzée, W. Siu, G. Gademann, P. Johnsson, M. Lucchini, R. R. Lucchese and M. J. J. Vrakking, Physical Review A 84, 051404 (2011).

16. A. Rouzée, F. Kelkensberg, W. K. Siu, G. Gademann, R. R. Lucchese and M. J. J. Vrakking, Journal of Physics B: Atomic, Molecular and Optical Physics 45, 074016 (2012).

17. A. Rouzée, A. G. Harvey, F. Kelkensberg, D. Brambila, W. K. Siu, G. Gademann, O. Smirnova and M. J. J. Vrakking, Journal of Physics B: Atomic, Molecular and Optical Physics 47, 124017 (2014). 18. C. Marceau, V. Makhija, D. Platzer, A. Y. Naumov, P. B. Corkum, A. Stolow, D. M. Villeneuve and P. Hockett, Physical Review Letters 119, 083401 (2017).

19. E. T. Karamatskos, S. Raabe, T. Mullins, A. Trabattoni, P. Stammer, G. Goldsztejn, R. R. Johansen, K. Dlugolecki, H. Stapelfeldt, M. J. J. Vrakking, S. Trippel, A. Rouzee and J. Kupper, Nature Communications 10, (2019).

20. M. D. Poulsen, E. Péronne, H. Stapelfeldt, C. Z. Bisgaard, S. S. Viftrup, E. Hamilton and T. Seideman, The Journal of Chemical Physics 121, 783 (2004).

21. K. F. Lee, D. M. Villeneuve, P. B. Corkum, A. Stolow and J. G. Underwood, Physical Review Letters 97, 173001 (2006).

22. X. Ren, V. Makhija and V. Kumarappan, Physical Review Letters 112, 173602 (2014).

23. K. Lin, I. Tutunnikov, J. J. Qiang, J. Y. Ma, Q. Y. Song, Q. Y. Ji, W. B. Zhang, H. X. Li, F. H. Sun, X. C. Gong, H. Li, P. F. Lu, H. P. Zeng, Y. Prior, I. S. Averbukh and J. Wu, Nature Communications 9, 9 (2018).

24. L. Holmegaard, J. L. Hansen, L. Kalhoj, S. Louise Kragh, H. Stapelfeldt, F. Filsinger, J. Küpper, G. Meijer, D. Dimitrovski, M. Abu-samha, C. P. J. Martiny and L. Bojer Madsen, Nat. Phys. 6, 428 (2010). 
25. L. Holmegaard, J. H. Nielsen, I. Nevo, H. Stapelfeldt, F. Filsinger, J. Küpper and G. Meijer, Physical Review Letters 102, 023001 (2009).

26. L. Holmegaard, J. L. Hansen, L. Kalhoj, S. Louise Kragh, H. Stapelfeldt, F. Filsinger, J. Küpper, G. Meijer, D. Dimitrovski, M. Abu-samha, C. P. J. Martiny and L. Bojer Madsen, Nature Physics 6, 428 (2010).

27. J. L. Hansen, H. Stapelfeldt, D. Dimitrovski, M. Abu-samha, C. P. J. Martiny and L. B. Madsen, Physical Review Letters 106, 073001 (2011).

28. D. Dimitrovski, J. Maurer, H. Stapelfeldt and L. B. Madsen, Physical Review Letters 113, 103005 (2014).

29. J. Maurer, D. Dimitrovski, L. Christensen, L. B. Madsen and H. Stapelfeldt, Physical Review Letters 109, 123001 (2012).

30. D. Rolles, R. Boll, M. Adolph, A. Aquila, C. Bostedt, J. D. Bozek, H. N. Chapman, R. Coffee, N. Coppola, P. Decleva, T. Delmas, S. W. Epp, B. Erk, F. Filsinger, L. Foucar, L. Gumprecht, A. Hömke, T. Gorkhover, L. Holmegaard, P. Johnsson, C. Kaiser, F. Krasniqi, K.-U. Kühnel, J. Maurer, M. Messerschmidt, R. Moshammer, W. Quevedo, I. Rajkovic, A. Rouzée, B. Rudek, I. Schlichting, C. Schmidt, S. Schorb, C. D. Schröter, J. Schulz, H. Stapelfeldt, M. Stener, S. Stern, S. Techert, J. Thøgersen, M. J. J. Vrakking, A. Rudenko, J. Küpper and J. Ullrich, Journal of Physics B: Atomic, Molecular and Optical Physics 47, 124035 (2014).

31. V. Kumarappan, C. Z. Bisgaard, S. S. Viftrup, L. Holmegaard and H. Stapelfeldt, The Journal of Chemical Physics 125, 194309 (2006).

32. U. Even, J. Jortner, D. Noy, N. Lavie and C. Cossart-Magos, J. Chem. Phys. 112, 8068 (2000). 33. A. S. Chatterley, E. T. Karamatskos, C. Schouder, L. Christiansen, A. V. Jørgensen, T. Mullins, J. Küpper and H. Stapelfeldt, The Journal of Chemical Physics 148, 221105 (2018).

34. A. S. Chatterley, C. Schouder, L. Christiansen, B. Shepperson, M. H. Rasmussen and H. Stapelfeldt, Nature Communications 10, 133 (2019). 
35. V. Dribinski, A. Ossadtchi, V. A. Mandelshtam and H. Reisler, Review of Scientific Instruments 73, 2634 (2002).

36. A. P. P. Natalense and R. R. Lucchese, Journal of Chemical Physics 111, 5344 (1999).

37. F. A. Gianturco, R. R. Lucchese and N. Sanna, Journal of Chemical Physics 100, 6464 (1994).

38. M. S. Gordon and M. W. Schmidt, Advances in electronic structure theory: GAMESS a decade later. In Theory and Applications of Computational Chemistry: the first forty years, Dykstra, C. E.; Frenking, G.; Kim, K. S.; Scuseria, G. E., Eds. Elsevier: Amsterdam, 2005; pp 1167.

39. M. W. Schmidt, K. K. Baldridge, J. A. Boatz, S. T. Elbert, M. S. Gordon, J. H. Jensen, S. Koseki, N. Matsunaga, K. A. Nguyen, S. Su, T. L. Windus, M. Dupuis and J. A. Montgomery, J. Comput. Chem. 14, 1347 (1993).

40. E. Drougas, J. G. Philis and A. M. Kosmas, Journal of Molecular Structure-Theochem 758, 17 (2006).

41. T. Hashimoto, H. Nakano and K. Hirao, Journal of Chemical Physics 104, 6244 (1996).

42. M. Rubio, M. Merchan, E. Orti and B. O. Roos, Chemical Physics 179, 395 (1994).

43. P. Hockett 2019, 10.17605/OSF.IO/PSJXT, https://osf.io/psjxt/wiki/aniline/.

44. P. Hockett 2019, DOI: 10.5281/zenodo.3612509

https://phockett.github.io/ePSdata/aniline/aniline wf $0.1-10.1 \mathrm{eV}$ orb33 A2.html

45. P. Hockett 2019, DOI: 10.5281/zenodo.3960685,

https://phockett.github.io/ePSdata/naphthalene/naphthalene wf 1.0-30.1eV orb33-45 S1.html.

46. K. L. Reid, Annual Review of Physical Chemistry 54, 397 (2003).

47. Z. Qu, Z. Qin, X. Zheng, H. Wang, G. Yao, X. Zhang and Z. Cui, Spectrochimica Acta Part A: Molecular and Biomolecular Spectroscopy 173, 432 (2017).

48. J. O. F. Thompson, B. Flinn, L. Whalley and K. L. Reid, unpublished data

49. M. Stockburger, H. Gattermann and W. Klusmann, J. Chem. Phys. 63, 4519 (1975).

50. J. O. F. Thompson, C. Amarasinghe, C. D. Foley and A. G. Suits, J. Chem. Phys. 147, 013913 (2017). 
51. J. O. F. Thompson, C. Amarasinghe, C. D. Foley, N. Rombes, Z. Gao, S. N. Vogels, S. Y. T. van de Meerakker and A. G. Suits, J. Chem. Phys. 147, 074201 (2017).

52. M. C. R. Cockett, H. Ozeki, K. Okuyama and K. Kimura, Journal of Chemical Physics 98, 7763 (1993).

53. M. Staniforth, S. Daly, K. L. Reid and I. Powis, The Journal of Chemical Physics 139, 064304 (2013).

54. S. M. Bellm, J. A. Davies, P. T. Whiteside, J. Guo, I. Powis and K. L. Reid, J. Chem. Phys. 122, 224306 (2005).

55. H. Stapelfeldt and T. Seideman, Rev. Mod. Phys. 75, 543 (2003).

56. T. Mullins, E. T. Karamatskos, J. Wiese, J. Onvlee, A. Rouzée, A. Yachmenev, S. Trippel and J. Küpper, submitted for publication (2020), https://arxiv.org/abs/2009.08157. 